\title{
CD44 expression in Dentigerous cyst, Radicular cyst and Ameloblastoma, by immunohistochemical analysis.
}

\author{
Dr. Sahana Srinath ${ }^{1}$, Dr. Asha Iyengar ${ }^{2}$, Dr. Vijaya Mysorekar ${ }^{3}$. \\ ${ }^{1}$ (Department of Oral Pathology and Microbiology, Government Dental College, Bangalore, Karnataka, India.) \\ 2 (Department of Oral medicine and Radiology, R. V. Dental College, Bangalore, Karnataka, India.) \\ 3 (Department of Pathology, M.S. Ramaiah Medical College, Bangalore, Karnataka, India.)
}

\begin{abstract}
The expression of cell adhesion molecule CD44s was analyzed immunohistochemically in 20 odontogenic lesions. CD44s was detected in almost all the cells on the cell membrane in basal and parabasal cells of the odontogenic cystic lining of the dentigerous cysts and the radicular cysts sparing the superficial layer of the cystic lining. CD44s was expressed on the cell membrane of the peripheral columnar cells and in the stellate cells, but were very negligible in the keratinizing areas.
\end{abstract}

Key words: Ameloblastoma, CD44, Dentigerous cyst, immunohistochemistry, Radicular cyst.

\section{Introduction}

Odontogenic epithelium is responsible for tooth development under physiologic conditions and can give rise to tumors or cysts in the jaws [1-3].Periapical cysts are the most common odontogenic cystic lesions of inflammatory origin. These cysts are found at the root apices of involved teeth, and are defined as odontogenic periapical cysts of inflammatory origin that are preceded by a chronic periapical granuloma[4].Dentigerous cysts are benign, non-inflammatory odontogenic cysts that are thought to be developmental in origin. Dentigerous cysts are the second most common odontogenic cysts after those related to the roots of the teeth (periapical cysts).A dentigerous cyst is formed by the hydrostatic force exerted by the accumulation of fluid between reduced enamel epithelium and the tooth crown of unerupted teeth [5]. Ameloblastoma is most commonly encountered tumour arising from odontogenic epithelium and is characterized by a benign but a locally invasive behavior with a high tendency to recur. Histologically ameloblastoma shows considerable variation, including follicular, plexiform, acanthomatous, granular cell, basal cell and desmoplastic type. These epithelial odontogenic tumors histologically resembles the epithelial odontogenic apparatus, such as enamel organ or dental lamina, in some respects; however, the detailed mechanisms of oncogenesis and cytodifferentiation remains unknown [6].

Cell to cell adhesion is indispensable for the regulation of cellular behavior, and a number of cell adhesion molecules mediating many kinds of adhesion have been identified[7]. CD44 is a family of cell surface glycoproteins that play a role in cell-cell and cell-extracellular matrix adhesion and interactions. CD44 gene contains at least 20 exons [8]. CD44 functions as a principle receptor for hyaluronic acid, a major glycosaminoglycan of the extracellular matrix. Altered CD44 expression especially that of CD44v, has been detected in various neoplasms, and CD44 is thought to play a part in tumor cell differentiation, progression and metastasis [8].

Recently, expression of CD44 has been studied in tooth germ tissues, and these molecules might have specific roles in cell interactions during tooth development.QIN YU in his study showed CD44 isoforms are expressed by proliferating epithelia at numerous sites of epithelial-mesenchymal interaction in the developing embryo; these sites include dental, nasal, aural, limb and hair follicle epithelium and concluded that CD44 expression is associated with dental epithelial derivatives including dental lamina, inner dental epithelium and ameloblasts; it is absent or low in amount in early dental mesenchyme, dental papilla, and dental follicle. CD44 is also absent from the developing layer of odontoblasts [9].

In the present study, immunohistochemical expression of CD44s was examined in Ameloblastoma, Dentigerous cyst and Radicular cyst to find out the role of this adhesion molecule in epithelial odontogenic lesions.

\section{Material And Methods}

20 cases of previously diagnosed odontogenic lesions, paraffin embedded, [5 each of follicular and plexiform Ameloblastoma, Dentigerous cyst and Radicular cyst] were obtained from the archives' of the department of oral pathology, Government dental college, Bangalore, India, and were reviewed and assessed according to the established criteria. 


\subsection{Immunohistochemistry:}

Four micrometer thick paraffin sections were prepared from the tissue block for the immunohistochemical staining. Antigen retrieval was performed by immersing the sections in a citrate buffer (pH6.0) for $10 \mathrm{~min}$. Endogenous peroxidases were blocked by incubating the sections with hydrogen peroxide for 5 min. Sections were then incubated with mono-clonal antibodies against CD44-s-2 kit (lab India instruments) (dilution 1: 100) were applied to the tissue sections and incubated overnight at $4^{\circ} \mathrm{C}$. Secondary biotinylated antibody and streptavidin HRP conjugated complex were applied for 60 and 30 minutes, respectively. After washing with the buffer, the chromogen diaminobenzidine was applied for 5 minutes.

After washing the slides were counterstained with Mayer's hematoxylin. Negative controls were performed by omitting the incubation of the primary antibody. Sections of normal oral mucosa were stained in parallel runs as positive controls. Additionally plasma cells present within each section served as internal controls.

\subsection{Immunohistochemical Evaluation}

CD44 immunoreactivity was evaluated by scanning the slides at medium and high magnifications (Olympus Optical Company). Five areas were randomly selected which were rich in lesional cells in the cystic linings of dentigerous cysts and radicular cyst, and ameloblastoma. The immunoreactivity was semi quantitatively scored using a 4-grade scoring: 0 (no staining); 1 (low), 1-10\% positive cells; 2 (intermediate), $11-50 \%$ positive cells; 3 (high), $>50 \%$ positive cells.

\section{Results}

The immunohistochemical reactivity for CD44 was of grade 3 and was detected in the plasma membrane of the cells in stellate like cells and peripheral columnar and cuboidal cells in ameloblastoma"Fig.1". The reactivity was negative /mild (0-1) in the acanthomatous areas.

The immunohistochemical reactivity for CD44 in both dentigerous cyst and radicular cysts were found to be similar to each other. Both the basal and parabasal cells showed high positivity for CD44 pericellularly whereas the superficial cells were negative "Fig. 2 and 3". The rushton bodies in this study was found to be blackish brown in colour and refractile "Fig. 4"

The chronic inflammatory cells in all the lesions showed high positivity for CD44 but the acute inflammatory cells were negative "Fig. 5 ".

\section{Figures}

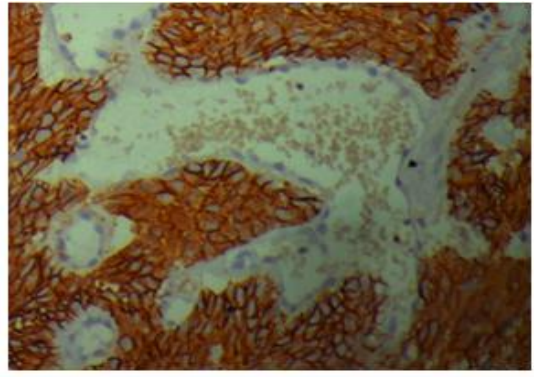

Figure 1. Ameloblastoma, plexiform ameloblastoma showing CD44 reactivity on the cell membrane of most of the tumor cells of ameloblastoma.

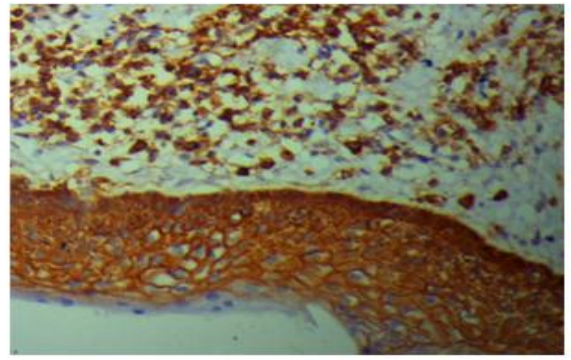

Figure 2: Dentigerous cyst with inflammation: reactivity was found on the cell membrane of the basal and parabasal cells of the cystic lining sparing the superficial layer. 

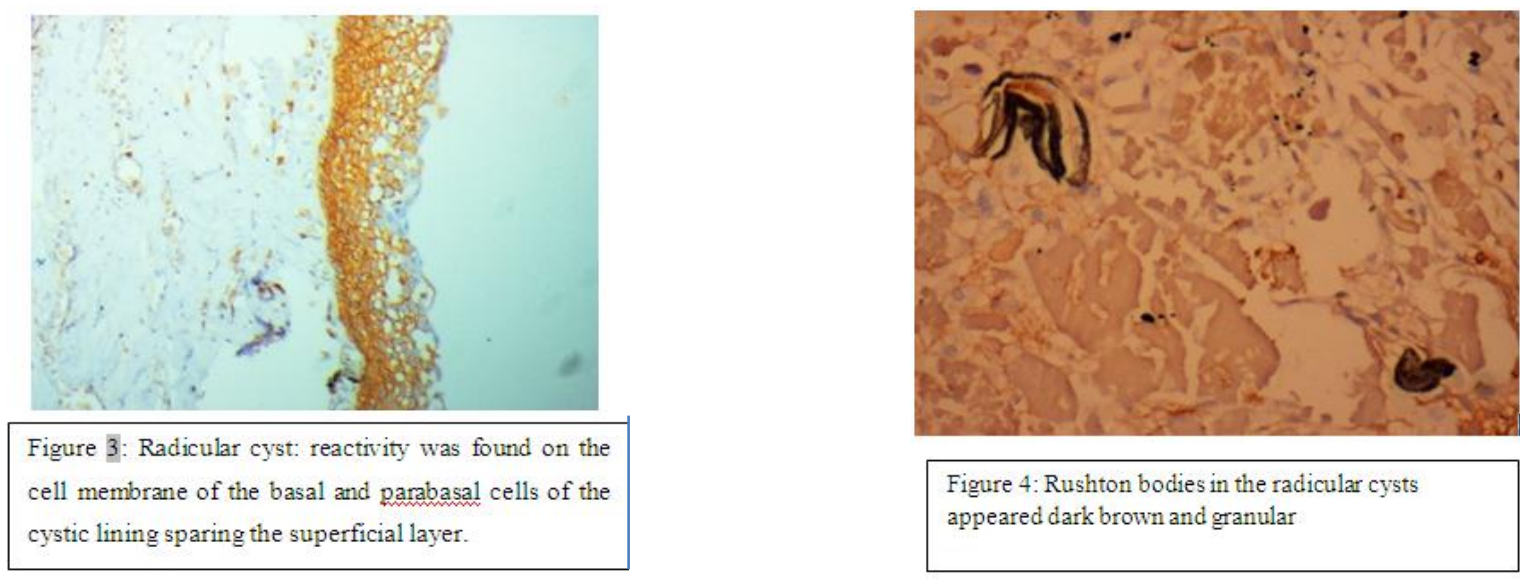

Figure 4 : Rushton bodies in the radicular cysts appeared dark brown and granular
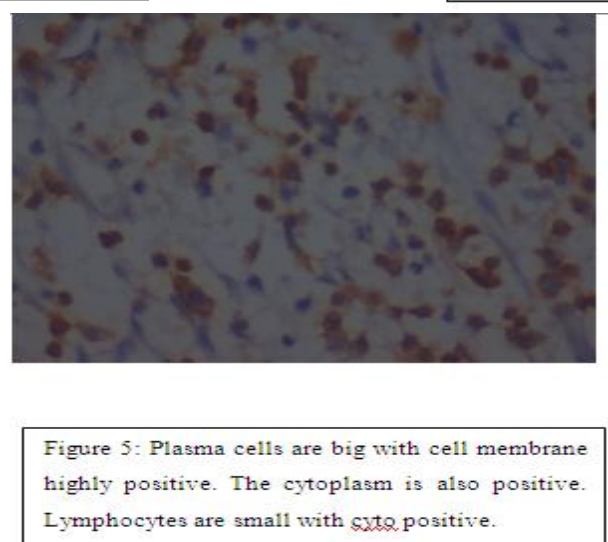

\section{Discussion:}

The $C D 44$ gene is unique for all the various isoforms of the protein. It was mapped in chromosome 11 p13 and includes at least 19 exons. Exons 1-5 and 16-19 are spliced together to form transcript, known as CD44s isoform, predominantly presented in haematopoietic tissues. Exons 6-15 are variable exons, alternatively spliced in insertion site between 5 and 16, generating various isoforms of molecule [10, 11].

CD44 is expressed in a wide variety of cell types, including hematopoietic cells, fibroblasts, macrophages, epithelial cells, muscle cells and glial cells [8], this molecule functions as a major receptor for hyaluronic acid, and is involved in adhesion, movement and activation of normal and transformed cells changes in CD44 expression are associated with progression and metastasis in a variety of human tumors [12].

Expression of CD44 has been recognized in dental epithelial structures of tooth germs, suggesting that these molecules play a role in morphogenesis and differentiation during tooth development [13].

Tooth development involves a series of reciprocal inductions between dental epithelium and neural crest derived mesenchymal cells. These processes start with thickening of the oral epithelium and formation of dental lamina at discrete sites within this epithelium. This is followed by invagination of the dental lamina into underlying mesenchyme and cytodifferentiation through the well-characterized bud, cap and bell stages of tooth development. Wheatley et al. (1993) noted a strong reaction with antibody to CD44 in early odontogenic placodes in the Embryonic day (E) 10.5 mouse embryo [14].

QIN YU detected a strong signal for CD44 at discrete sites within oral epithelium where the dental lamina is formed. At the early bud stage, expression of CD44 is restricted to the dental lamina and invaginating dental epithelial cells. This expression pattern is maintained until the late bud stage. In the 15-day embryo, the first molar tooth germ is at the cap stage, at which time CD44 is expressed strongly in the dental lamina and inner dental epithelium. As tooth development reaches the bell stage, CD44 is expressed at a moderate level in the differentiating ameloblast and at high levels in the adjacent stratum intermedium but is absent from most of the remaining mesenchyme and the developing odontoblasts. At these stages of tooth development most of the associated mesenchymal cells, including condensed mesenchyme adjacent to the invaginating dental lamina and in the forming dental papilla exhibit negligible amounts of CD44. QIN YU conclude from these results that CD44 expression is associated with dental epithelial derivatives including dental lamina, inner dental epithelium and ameloblasts; it is absent or low in amount in early dental mesenchyme, dental papilla, and dental follicle. CD44 is also absent from the developing layer of odontoblasts [9]. 
In the present study, CD44 was widely expressed in tumour cells of ameloblastoma except in the highly differentiated acanthomatous area where it was very mildly expressed. In the radicular cyst and dentigerous cyst the CD44 was expressed in basal and parabasal layer of the epithelium. The superficial cells were negative which could be because of increased maturity. These results suggest that CD44 might be involved not only in cell adhesion but also in tumour progression and are found in active cell in the odontogenic epithelial lesions.

\section{Conclusion}

In conclusion these observations suggest that these cell adhesion molecules are strongly expressed in the active cells than in the differentiated cells and might be involved in the tumor progression. Immunohistochemical staining of odontogenic lesions for CD44s may be useful in detecting the active cells in the odontogenic lesions and to predict the tumor progression.

We thank Dr. Srinath S K for his assistance.

\section{Acknowledgements}

\section{References}

[1]. Shear M, Developmental odontogenic cysts: an update. J Oral Pathol Med 1994; 23: 1-11.

[2]. Melrose R J. Bening epithelial odontogenic tumors. Semin Diagn Pathol 1999;16: 271-87.

[3]. Eversole L R. Malignant epithelial odontogenic tumors. Semin Diagn Pathol 1999; a16:317-24.

[4]. Shear M. Radicular and residual cysts. Cysts of the Oral Region, 3rd ed. Bristol UK: Wright. 1992:136-162.

[5]. Som PM, Curtin HD. Head and neck imaging. Mosby Inc. (2003) ISBN:0323009425.

[6]. Kramer IRH, Pindborg JJ and Shear M. WHO histological typing of odontogenic tumors. Springer- Verlag, Berlin. 1992; 11-6.

[7]. Gumbiner BM. Cell adhesion: the molecular basis of tissue architecture and morphogenesis. Cell 1996;84:345-57.

[8]. Naor D, Sionov R V and Ish-Shalom D. CD44: structure, function, and association with the malignant process. Adv Cancer Res 1997; 71:241-319.

[9]. Qin yu and bryan p. Toole. Common Pattern of CD44 Isoforms is Expressed in Morphogenetically Active Epithelia. Developmental dynamics 1997;208:1-10.

[10]. Hirano H, Screaton GR, Bell MV, Jackson DG, Bell JI, Hodes RJ. CD44 isoform expression mediated by alternative splicing: tissue-specific regulation in mice. Int Immunol 1994; 6: 49-59.

[11]. Van Weering DH, Baas PD, Bos JL. A PCR-based method for the analysis of human CD44 splice products. PCR Methods Appl 1993; 3: 100-6.

[12]. Ue T, Yokozaki H, Kagai K et. Al. Reduced expression of the CD44 variant exons in oral suamous cell carcinoma and its relationship to metastasis. J Oral Pathol Med 1998;27:197-201.

[13]. Yu Q and Toole B P. Common pattern of CD44 isoforms is expressed in morphogenetically active epithelia. Dev Dyn 1997; 208: 110 .

[14]. Wheatley, S. C., Isacke, C. M. and Crossley, P. H. (1993). Restricted expression of the hyaluronan receptor, CD44, during postimplantation mouse embryogenesis suggests key roles in tissue formation and patterning. Development 119, 295-306. 